\title{
Visión de los estudiantes sobre una articulación curricular de tres cursos iniciales de ingeniería civil mecánica
}

\author{
Ana M. Moncada-Arce ${ }^{1}$, Enrique Ortiz-Vidal ${ }^{2}$ y Daniel E. Casagrande ${ }^{2^{*}}$ \\ (1) Universidad de Chile, Santiago-Chile (Correo-e: amoncada@uchile.cl) \\ (2) Instituto de Ciencias de la Ingeniería, Universidad de O'Higgins, Rancagua-Chile \\ (Correo-e: enrique.ortiz@uoh.cl; daniel.casagrande@uoh.cl) \\ ${ }^{*}$ Autor a quien debe ser dirigida la correspondencia.
}

Recibido Jun. 29, 2021; Aceptado Ago. 25, 2021; Versión final Oct. 24, 2021, Publicado Feb. 2022

\begin{abstract}
Resumen
El objetivo de este estudio es indagar las percepciones estudiantiles al participar de una experiencia de articulación curricular en tres cursos iniciales de ingeniería civil mecánica utilizando aprendizaje basado en proyecto. El proyecto consistió en el diseño y construcción de una armadura usando impresión 3D. Se identifican las oportunidades y tensiones al participar de la experiencia, y se proponen mejoras al diseño. Se recolectaron las percepciones y opiniones de 11 estudiantes de dos de los cursos intervenidos, en dos momentos del semestre, utilizando encuestas grupales e individuales. Se utilizó análisis de contenido para procesar la información recolectada. Los resultados son coherentes con la literatura en cuanto al incremento en la motivación, la significación de contenidos y la valoración de la experiencia. El mayor desafío identificado por los participantes fue el trabajo en equipo. Se concluye que para mejorar la experiencia de aprendizaje se propone aumentar las coevaluaciones y la capacitación específica del cuerpo docente.
\end{abstract}

Palabras clave: enseñanza de la ingeniería; articulación curricular; aprendizaje activo; aprendizaje basado en proyecto; investigación cualitativa

\section{Students' views on linking curricular content of three first-year courses of a mechanical engineering program}

\begin{abstract}
The objective of this study is to explore students' perceptions of a project-based learning intervention of three first-year courses in a Chilean mechanical engineering program. The project consisted of designing and building a truss by using 3D printing. Students' views regarding this learning experience are assessed to identify learning opportunities and challenges that may emerge while working on the project. Individual and group interviews and questionnaires were conducted twice per semester in two courses to gather the views of 11 students. Collected data was processed by content analysis. The results showed higher motivation and content significance and increased experience value. These results are in agreement with findings reported in the literature. Teamwork was the biggest challenge identified by the participants. It is concluded that the learning experience can be improved by including frequent co-evaluations throughout the experience and the training of instructors on collaborative work.
\end{abstract}

Keywords: engineering teaching; content linkage; active learning; project-based; qualitative research 


\section{INTRODUCCIÓN}

La enseñanza de la ingeniería necesita ser orientada a responder a los desafíos actuales de la sociedad. Los cambios en el mercado laboral y los rápidos avances en el conocimiento científico y tecnológico presentan una demanda por formar profesionales que sean capaces de adatarse a estos cambios y que respondan eficazmente a las demandas crecientes de una sociedad cada vez más compleja. Estos desafíos, originados principalmente por el vertiginoso avance tecnológico, han sido sistematizados en iniciativas como los 17 Objetivos de Desarrollo Sostenible de las Naciones Unidas y también a través del laboratorio de ideas sin fines de lucro The Millenium Project. Para responder a estos desafíos, la formación de profesionales debe incluir además del conocimiento técnico de su área, espacios de aprendizaje que faciliten el desarrollo de competencias que les permitan poner en práctica estos conocimientos, de manera creativa, crítica y en contextos desafiantes y multidisciplinarios (Chua, 2014). Las habilidades que se fomenten durante la carrera de ingeniería permitirán a los estudiantes seguir aprendiendo a lo largo de la vida (Heitmann, 2005). Entre las habilidades necesarias están las relacionadas a las habilidades de innovación y aprendizaje, es decir, creatividad, pensamiento crítico, comunicación y colaboración (Framework for 21st Century Learning, 2021).

Para lograr el desarrollo de las habilidades y competencias descritas es necesario que la enseñanza sea adecuada, brindando espacios pertinentes para practicarlas y perfeccionarlas. Un avance en ese sentido es el reconocimiento e incorporación de competencias genéricas en los programas educativos como declara en el proyecto Tuning América Latina (Beneitone y Esqueteni, 2007). Este proyecto define las competencias genéricas como aquellas comunes a todas las profesiones y que permiten tener herramientas para desenvolverse en cualquier aspecto de la vida, como por ejemplo el trabajo en equipo. Otro avance que se puede señalar es la transición hacia metodologías centradas en el aprendizaje, lo que incluye tanto cambios estructurales, como el rediseño de currículos o cursos y cambios a pequeña escala, por ejemplo, la incorporación de estrategias de aprendizaje activo en las clases. Este segundo avance ha encontrado algunas barreras. Por ejemplo, la ausencia de conexiones significativas entre cursos percibidas por los estudiantes (Karkoub et al., 2020), dado que tradicionalmente los programas de ingeniería tienden a ser secuenciales y fragmentados por contenidos y áreas disciplinares (Meyer y Marx, 2014).

Una segunda barrera, es la ausencia de aplicaciones significativas de los contenidos presentes en el currículo de forma regular a lo largo del programa. Según Gomes (2002) las aplicaciones significativas tienden a ocurrir al final del programa de estudios en forma de tesis y/o diseños. Las barreras citadas se han relacionado con algunos aspectos negativos o no deseados en la formación de ingenieros. Por ejemplo, Gomes (2002) sugiere que el foco en las áreas disciplinares y las conexiones poco explícitas tienden a una disposición al aprendizaje memorístico. Además, Froyd y Ohland (2005) indican que se ha cuestionado en repetidas ocasiones la factibilidad de que los cursos que deliberadamente compartimentan los contenidos de la malla curricular puedan proporcionar actividades de aprendizaje que estimulen, animen y permitan a los estudiantes estructurar su conocimiento dentro de los límites disciplinarios y de los cursos. Estos autores sugieren que los entornos de aprendizaje que enfatizan las conexiones entre los cursos pueden mejorar el grado en que los estudiantes podrían transferir sus conocimientos (de matemática y ciencias) a los cursos de ingeniería.

Existe una falta de concordancia entre las competencias y habilidades necesarias para el ejercicio de la profesión de ingeniería, y las que el currículo y metodologías de enseñanza proporcionan a los futuros profesionales. La literatura revisada sugiere que la falta de concordancia se debe, entre otros factores, a la poca promoción de aplicaciones significativas (que integren contenidos y habilidades) a lo largo de la formación y a la pobre articulación de cursos e integración de contenidos (ver por ejemplo, Froyd y Ohland, 2005; Meyer y Marx, 2014). En Chile, no se encontraron reportes cualitativos de la evaluación de los factores mencionados y cómo estos influyen en la motivación de los estudiantes.

Una metodología que propicia el aprendizaje a través de aplicaciones significativas es el aprendizaje basado en proyectos (ABPro). En ABPro los estudiantes deben desarrollar una solución pertinente a un problema considerando las restricciones/limitaciones del contexto. Esta metodología se focaliza en el proceso de aprendizaje y busca formar aprendices activos, autónomos, estratégicos, reflexivos, cooperativos y responsables (Chua, 2014). También ha mostrado tener efectos positivos en la motivación, cambiando las actitudes de los estudiantes, promoviendo la interacción y fomentando la autonomía (ver, Baepler y Walker, 2014; Dori y Belcher, 2005; Vega et al., 2014). Un factor clave en la implementación del ABPro es el rol docente. Por ejemplo, el docente debe encontrar proyectos en los que la brecha en el conocimiento (lo que se sabe y lo que se necesita saber para completar el proyecto) sea manejable por los estudiantes, de tal forma que conduzca al estudio autónomo y además crear el clima adecuado para conducir a los aprendizajes deseados. El ABPro ha sido utilizado extensamente en la educación en ingeniería (Christie y de Graaff, 2017), aunque con algunos desafíos en su implementación; entre ellos, la escasa o nula experiencia de los estudiantes en este tipo de actividades y su predilección por estructuras tradicionales de aprendizaje (Chua, 2014, Gratchev y Jeng, 2018), la falta de habilidades para trabajar colaborativamente (Le et al., 2018). Adicionalmente, mayor demanda de tiempo para la preparación, organización y administración de los proyectos puede también ser una limitante (Frank et al., 2003, Fuertes et al., 2015, Mesa et al., 2008). 
En el ABPro, tradicionalmente, se promueve el trabajo en equipo. Torrelles (2011) en su trabajo Eina d'avaluació de la competència de treball en equip indica que el trabajo en equipo es una competencia compleja que supone "la disposición personal y la colaboración con otros en la realización de actividades para lograr objetivos comunes, realizando actividades de intercambio de información, asignación de responsabilidades, resolución de conflictos, y una contribución a la mejora y desarrollo colectivo" (citado por Herrera et al., 2017, p. 50), por lo que podemos identificar algunas dimensiones claves como: coordinación y cooperación, resolución de conflictos, comunicación, y establecimiento de objetivos comunes. Esas dimensiones están de acuerdo con lo establecido en la literatura (Soria-Barreto y Cleveland-Slimming, 2020; Torrelles et al., 2011). Se destaca que en el trabajo en equipo coexisten lo personal y lo colectivo, justamente como sucede en el aprendizaje. La teoría socioconstructivista del aprendizaje propone una naturaleza sociocultural del aprendizaje, esto quiere decir que trabajar con otras personas es una componente crítica del proceso de construcción del conocimiento (Moore, 2011).

Esta manera de entender el aprendizaje considera que el mundo social del individuo es un elemento primordial para este proceso, lo que ha llevado a algunos autores como Wertsch (1991), a proponer que todas las funciones mentales superiores se originan como el resultado directo de la interacción entre individuos, lo que significa que el aprendizaje ocurre en un plano interpsicológico (entre personas) antes de que se internalice en un plano personal e intrapsicológico (dentro del individuo). Además de los aspectos cognitivos mencionados, el desarrollo de la competencia de trabajo en equipo busca que los estudiantes interioricen actitudes como la transparencia, constancia, compromiso y respeto (Herrera et al., 2017). También busca que los estudiantes desarrollen y practiquen habilidades como la argumentación, toma de decisiones, resolución de conflictos, negociación, liderazgo, entre otras. En Chile, Herrera et al. (2017) realizaron un diagnóstico del trabajo en equipo en los programas de ingeniería, encontrando que existen oportunidades de trabajar en equipo con estudiantes de la misma carrera, pero no con otras.

Este trabajo es una investigación cualitativa que analiza las percepciones de estudiantes chilenos que participaron en un proyecto de articulación en tres cursos de primer año de la especialidad de Ingeniería Civil Mecánica. El proyecto de articulación surge de la reflexión y mejora de distintas actividades puestas en práctica por los docentes participantes y busca brindar oportunidades de integración de conocimientos y desarrollo de habilidades genéricas.

\section{METODOLOGÍA}

Esta investigación es de carácter cualitativo y se intervinieron tres cursos tradicionales de primer año de especialidad del programa de Ingeniería Civil Mecánica. La intervención consistió en el desarrollo de un proyecto enfocado en el diseño, análisis y construcción de una armadura; en el que los cursos actuaban como áreas de una empresa o consultoras de ingeniería. Estudiantes de dos de los cursos intervenidos participaron del estudio.

\section{Contexto de la intervención}

Los cursos intervenidos fueron Mecánica Estática y Dinámica (MED) y Dibujo Mecánico (DM) del tercer semestre del programa de la carrera y Mecánica de Sólidos (MS) correspondiente al cuarto semestre del programa, i.e. todos del primer año de la especialidad. El curso MED contó con 11 estudiantes, 3 de los cuales hacían en el curso por segunda vez. El curso DM tuvo 8 estudiantes, de los cuales 1 estudiante hacía el curso por segunda vez. Por último, el curso MS tuvo 3 estudiantes, todos inscritos por primera vez. Todos los estudiantes de los cursos intervenidos participaron del proyecto, haciendo un total de 17; 5 de ellos tomaban simultáneamente MED y DM.

La experiencia de articulación consideró la conformación de equipos de trabajo en cada curso, cada equipo asumiendo un rol específico en el proyecto. Para establecer el tamaño de los equipos, en cada curso se consideró la carga de trabajo para lograr los objetivos de aprendizaje propios de cada asignatura y la facilidad de comunicación entre los cursos. Así, el proyecto estuvo liderado por los equipos del curso MED donde se formaron 4 equipos de trabajo ( 2 y 3 personas), en DM trabajaron en pares (4 equipos) y en MS, dado que el curso solo tiene 3 estudiantes inscritos, el trabajo fue individual. Los equipos MED se desempeñaban como el área de Ingeniería de una empresa, los equipos DM como el área de diseño asistido por computadora (área CAD) de la misma empresa, y los estudiantes de MS como consultoras de ingeniería. Cada equipo MED articulaba el desarrollo del proyecto con un equipo DM y un estudiante MS. Los 5 estudiantes inscritos en MED y DM, simultáneamente, participaron con doble rol en el proyecto; cabe señalar que esos estudiantes inscribieron ambos cursos por primera vez y fueron distribuidos de tal forma que todos los equipos MED y DM tuvieron la presencia de al menos uno de ellos. 
Por último, el equipo docente que participó en el proyecto no tenía experiencia en el diseño ni implementación de ABPro. Por esta razón, se destinó tiempo para la capacitación y discusión de esta metodología y sus implicancias para la docencia (Mesa et al., 2008). Por ejemplo, al mismo tiempo que se diseñaba el proyecto, se indagó sobre cómo orientar el trabajo colaborativo en los estudiantes. Esto condujo al diseño de un taller inicial para el curso de MED. Además, durante la implementación del proyecto, los docentes se reunieron de manera sistemática para responder a las necesidades emergentes de los distintos equipos y cuidar el logro de los objetivos propuestos. Los resultados de aprendizaje esperados de cada curso se presentan en la tabla 1; en la que también se indica cuáles de ellos fueron abordados durante el desarrollo del proyecto.

Tabla 1. Productos solicitados según los cursos y las etapas del proyecto.

\begin{tabular}{|l|l|c|}
\hline \multirow{2}{*}{ Curso } & Resultado de aprendizaje & $\begin{array}{l}\text { Abordaje en } \\
\text { el proyecto }\end{array}$ \\
\hline $\begin{array}{l}\text { Mecánica estática } \\
\text { y dinámica (MED) }\end{array}$ & $\begin{array}{l}\text { Analiza estructuras isostáticas y mecanismos simples sometidos a cargas } \\
\text { estáticas }\end{array}$ & $\mathrm{X}$ \\
\cline { 2 - 3 } & $\begin{array}{l}\text { Maneja el análisis cinemático y el análisis dinámico de mecanismos planos y } \\
\text { espaciales }\end{array}$ & \\
\hline \multirow{2}{*}{$\begin{array}{l}\text { Diseño mecánico } \\
\text { (DM) }\end{array}$} & Revisa y analiza planos de conjunto, despiece y fabricación & $\mathrm{X}$ \\
\cline { 2 - 3 } & Reconoce partes y componentes de mecanismos & \\
\cline { 2 - 3 } & Reconoce especificaciones técnicas y sus aplicaciones & \\
\hline \multirow{2}{*}{$\begin{array}{l}\text { Mecánica de } \\
\text { sólidos (MS) }\end{array}$} & $\begin{array}{l}\text { Aplica principios y conceptos de la mecánica de sólidos y elasticidad lineal en } \\
\text { diversos problemas de la ingeniería mecánica, para el diseño de vigas, ejes y } \\
\text { otras componentes de máquinas y estructuras. }\end{array}$ & \\
\cline { 2 - 3 } & $\begin{array}{l}\text { Analiza varios fenómenos de falla en sólidos, en problemas de diseño de } \\
\text { mecanismos y estructuras simples, a fin de interpretar los resultados de los } \\
\text { modelos }\end{array}$ & $\mathrm{X}$ \\
\hline
\end{tabular}

\section{Participantes}

La participación de los estudiantes en el estudio fue voluntaria y se invitó a participar a todos los estudiantes de los tres cursos. En total, participaron 10 estudiantes, de los cuales 4 estaban inscritos sólo en MED, 1 sólo en DM y 5 en ambos cursos. Se señala que los estudiantes MS desarrollaron el proyecto de articulación, pero no participaron en el estudio.

\section{Descripción del proyecto}

Considerando los objetivos de aprendizaje de cada curso, se diseñó una experiencia de aprendizaje, a través de la realización de un proyecto, que consolide conceptos y herramientas de estática de cuerpo rígido, aplicación de principios y normas de dibujo mecánico, y los criterios de falla de resistencia de materiales. Además de aportar a las competencias técnicas de cada curso, el proyecto debía contribuir al desarrollo de competencias genéricas; por ejemplo, trabajo en equipo, gestión de proyectos y comunicación oral y escrita. Estas competencias incluyen ámbitos como la presentación de resultados, redacción de informes, coordinación, comunicación y resolución de conflictos. Así, el proyecto es una instancia de integración de conocimientos y desarrollo de competencias técnicas y genéricas. El proyecto consistió en el diseño, análisis y construcción de una estructura de armaduras a escala reducida empleando impresión 3D. Se presentaron dos alternativas de armaduras: un puente y un techo, en ambos casos se indicaron las dimensiones que debía cubrir la estructura, la escala del modelo a construir y las cargas que debía soportar.

El proyecto fue liderado por los estudiantes de MED y se articuló con estudiantes de DM y MS. Dado este liderazgo se desarrollaron en el curso de MED actividades donde se les entregaron herramientas para trabajar en equipo. Al iniciar el proyecto, se realizó un taller donde cada equipo organizó la forma de trabajar durante el semestre dejando plasmado un contrato con los acuerdos. En particular, cada equipo definió, de manera flexible, roles para sus integrantes; modos de comunicación y de reunión; el funcionamiento del equipo para la toma de decisiones y penalizaciones para integrantes que faltasen a los compromisos asumidos. El taller finalizó con la redacción y firma del contrato por parte de todos los integrantes del equipo. Además, en una de las evaluaciones de finalización del proyecto se incorporó la evaluación de pares o coevaluación, pues es una práctica que permite el desarrollo de la capacidad de pensamiento crítico y la regulación de los aprendizajes tanto individuales, como colectivos (Hanrahan e Isaacs, 2001). 


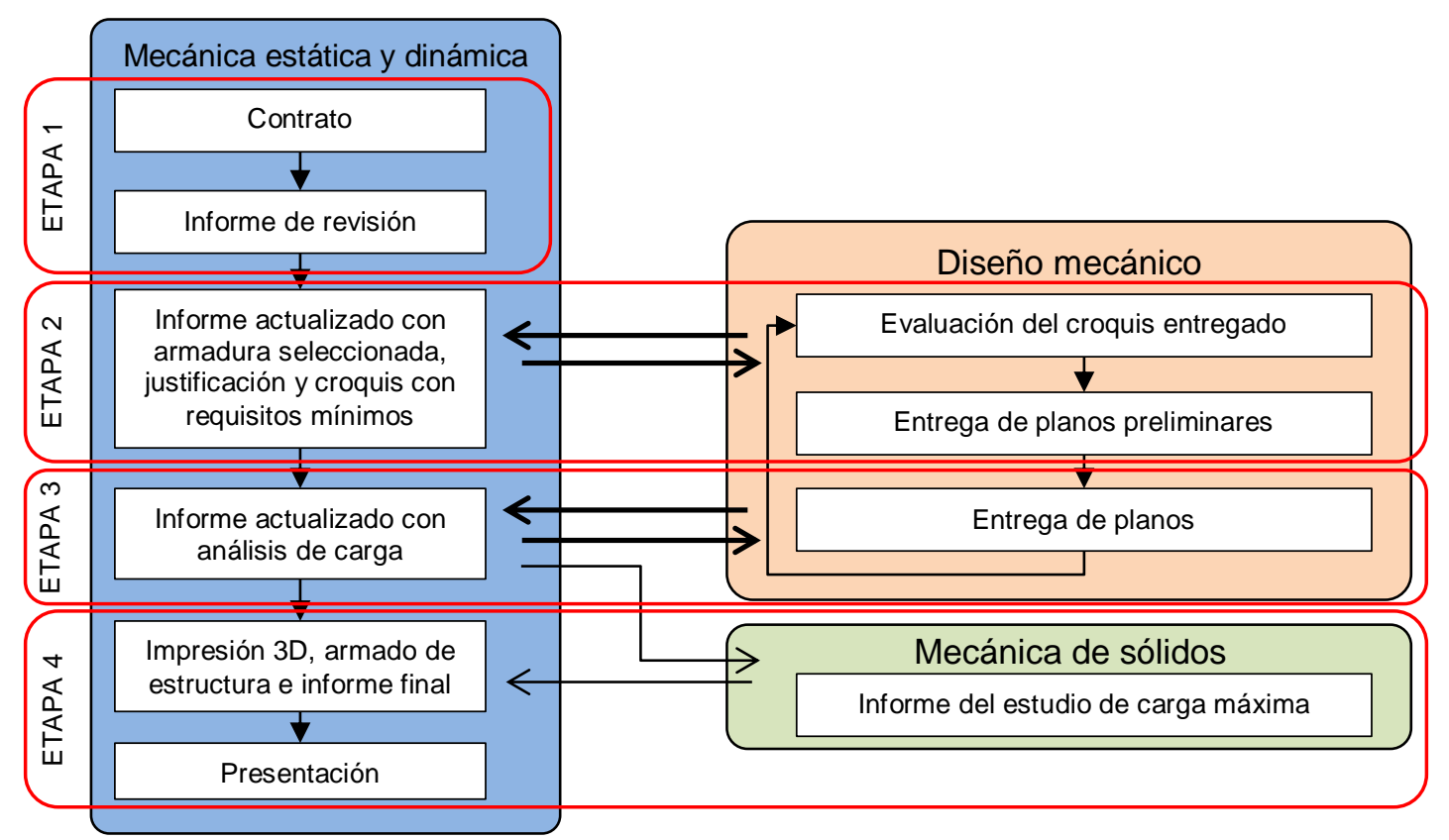

Fig. 1: Esquema de la intervención del proyecto de armadura a escala reducida.

Se definieron 4 etapas para organizar y monitorear el avance del proyecto y las interacciones entre estudiantes de los distintos cursos. Las etapas e interacciones están indicadas en el esquema presentado en la Figura 1. Para cada equipo de MED se asignó un equipo de DM y un estudiante de MS; es decir, cada equipo de MED contó con el diseño producido por el equipo de DM y el estudio de carga máxima realizado por el estudiante de MS. Recordemos que los roles de los equipos de MED, DM y MS son área de Ingeniería de una empresa, área de diseño asistido por computadora (área CAD) de la misma empresa, y consultora de ingeniería, respectivamente.

El proyecto inicia en la etapa 1 con una actividad guiada para los estudiantes de MED en la que cada equipo redactó su contrato, asignando roles, delimitando responsabilidades, canales de comunicación y penalizaciones por incumplimiento. La primera tarea técnica fue la realización de un informe de revisión de lo más avanzado en armaduras. Se entregaron consignas sobre el formato del informe para la redacción de informes de avance y se utilizaron pautas de cotejo, tanto para el contrato de trabajo en equipo como para los informes de avance. Para facilitar la gestión del trabajo en equipo y del proyecto se entregó un cronograma de entregas y de actividades que llevaría cada curso durante el semestre y se favoreció el uso de la herramienta Trello.

Durante la etapa 2 cada equipo de MED debió seleccionar un tipo de armadura, justificar la elección y realizar un croquis de la solución propuesta que incluya requerimientos mínimos. Al finalizar esta etapa sucede la primera interacción con el equipo de DM asignado, quienes reciben el croquis con los requerimientos y deben evaluar este documento, interactuar con el equipo de MED si fuese necesario y confeccionar los planos preliminares. Las relaciones entre equipos de distintos cursos se indican con flechas negras horizontales en la Figura 1, donde el tamaño de las flechas indica si hubo solo entrega de información (flechas pequeñas) o interacción entre los equipos (flechas grandes).

En la etapa 3, el equipo de MED debió realizar un estudio de carga propuesto por el equipo docente e interactuar con el área CAD (equipos de DM) que desarrolla los planos. El desarrollo de los planos se caracterizó por una discusión técnica en sesiones presenciales en el curso de DM. A modo de ejemplo, en cada sesión el profesor iniciaba sustentando el avance de un plano; seguidamente cada grupo hacía lo mismo frente a la clase y recibía retroalimentación de sus pares. La consigna fue que los comentarios debían incluir -por lo menos- un aspecto positivo, una crítica y un aspecto a mejorar. El profesor moderó las tres sesiones de discusión realizadas. Al finalizar la etapa 3, el informe del estudio de carga realizado por MED se envió al Estudio de ingenieros (estudiantes MS) para su revisión y realización de estudio de carga máxima soportada por la estructura. Durante la etapa 4, con los planos ya finalizados, se procede a la construcción de la armadura (impresión 3D y ensamble) e integración de toda la información en el informe final. La última etapa finalizó con una presentación abierta a la comunidad universitaria en la que cada equipo presentó su estructura, realizó el ensayo de carga y respondió los cuestionamientos de los asistentes (profesores y estudiantes). Se entregaron consignas para las presentaciones orales y rúbrica de evaluación. El monitoreo del proyecto se realizó a través de los productos indicados en la Tabla 2. 
Tabla 2. Productos solicitados según los cursos y las etapas del proyecto.

\begin{tabular}{|c|l|l|l|}
\hline Etapa & Equipo de MED (área de Ingeniería) & Equipo de DM (área CAD) & $\begin{array}{l}\text { Equipo de MS (consultora de } \\
\text { ingeniería) }\end{array}$ \\
\hline 1 & $\begin{array}{l}\text { Contrato de equipos } \\
\text { Informe de revisión de tecnología de } \\
\text { vanguardia en armaduras. }\end{array}$ & Sin entregas & Sin entregas \\
\hline 2 & $\begin{array}{l}\text { Informe actualizado que incluye la } \\
\text { estructura seleccionada, la } \\
\text { justificación de la elección, croquis y y } \\
\text { requerimientos mínimos. }\end{array}$ & $\begin{array}{l}\text { Planos preliminares en base a los } \\
\text { requerimientos mínimos } \\
\text { establecidos por el equipo de } \\
\text { MED. Se entregaron archivos en } \\
\text { formato editable y PDF. }\end{array}$ & Sin entregas \\
\hline 3 & $\begin{array}{l}\text { Informe actualizado que incluye el } \\
\text { estudio de carga de la estructura } \\
\text { seleccionada. }\end{array}$ & $\begin{array}{l}\text { Planos finales en base a la } \\
\text { interacción entre grupos. Se } \\
\text { entregaron archivos en formato } \\
\text { editable y PDF. }\end{array}$ & $\begin{array}{l}\text { Informe de estudio de carga } \\
\text { máxima soportada por la } \\
\text { informe de estudio de carga } \\
\text { de MED. }\end{array}$ \\
\hline 4 & $\begin{array}{l}\text { Informe completo, que incluye los } \\
\text { aspectos anteriores señalados e } \\
\text { incorpora el estudio de carga } \\
\text { máxima, planos y conclusiones. } \\
\text { Presentación a la comunidad. }\end{array}$ & Sin entregas & Sin entregas \\
\hline
\end{tabular}

\section{Objetivo del estudio y preguntas de investigación}

Este artículo presenta un estudio cualitativo cuya finalidad es conocer y comprender las oportunidades y desafíos que los estudiantes perciben, tanto individual como colectivamente, al participar de la experiencia de aprendizaje propuesta, que articula horizontalmente 3 cursos de primer año de especialidad de la carrera de Ingeniería Civil Mecánica. La experiencia de aprendizaje se basa en el desarrollo del proyecto descrito anteriormente, donde grupos de estudiantes de los diferentes cursos asumen roles específicos e interactúan entre ellos para el diseño, análisis y construcción de una estructura de armadura. Las preguntas iniciales de investigación que guían este estudio son: PI-1) ¿Qué aspectos rescatan los estudiantes de la incorporación del proyecto de armaduras en el currículo de los cursos? Y PI-2) ¿Cuáles son las principales dificultades que se presentan en la implementación del proyecto de armaduras?

\section{Métodos de investigación}

Este es un estudio cualitativo sobre las percepciones de los estudiantes al participar en un proyecto de articulación de 3 cursos de primer año de especialidad de la carrera de Ingeniería Civil Mecánica. Se utilizaron dos instrumentos de recolección de datos, 1) entrevistas grupales semiestructuradas y 2) cuestionarios individuales. Las entrevistas grupales permiten recolectar datos relacionados con actitudes, valores y opiniones de los participantes, dando flexibilidad para que los participantes puedan dar detalles en algún tema de su interés y contrastar opiniones durante la entrevista, mientras los cuestionarios individuales focalizan la recolección de información en temáticas específicas, permitiendo la comparación de las respuestas individuales. En este estudio durante las 1) entrevistas grupales semiestructuradas, se indagó sobre las oportunidades y desafíos que enfrentaron al participar en el proyecto de armadura. Los temas específicos que guiaron esta entrevista fueron: (i) los desafíos que enfrentaron en el curso y en el proyecto, (ii) los aprendizajes logrados gracias al proyecto, y (iii) los aspectos del curso que contribuyen a su formación profesional. Además, se les pidió realizar recomendaciones para mejorar el proyecto y aspectos en los que los propios estudiantes podrían mejorar su trabajo en el proyecto, y las preguntas de los 2) cuestionarios individuales, se focalizaron en el aprendizaje individual, sobre la experiencia y sobre el trabajo en equipo.

Las entrevistas y cuestionarios fueron de carácter voluntario y solo estudiantes de MED y DM respondieron a la invitación al estudio. Los datos fueron analizados utilizando análisis de contenido (Krippendorff, 2004). Las entrevistas grupales se realizaron 3 semanas antes de la presentación final del proyecto y se organizaron de forma que los estudiantes del mismo equipo no hicieran la entrevista juntos. Al inicio de la entrevista se le pidió a cada grupo que discutiera los temas presentados y que entregara sus conclusiones y comentarios de manera escrita. El cuestionario individual fue aplicado vía remota luego de la finalización del proyecto. La Tabla 3 presenta la cantidad de participantes por instrumento de recolección de datos. 
Tabla 3. Cantidad de participantes en la recolección de datos.

\begin{tabular}{|c|c|c|l|}
\hline \multirow{2}{*}{ Curso } & \multicolumn{2}{|c|}{ Cantidad de participantes } & \multirow{2}{*}{ Observaciones respecto al cuestionario individual } \\
\cline { 2 - 3 } & En entrevista grupal & $\begin{array}{c}\text { Que respondieron el } \\
\text { cuestionario individual }\end{array}$ & \\
\hline MED & 9 & 7 & $\begin{array}{l}\text { 4 participantes estaban inscritos solo en MED. } \\
\text { 3 participantes estaban inscritos en MED y DM. }\end{array}$ \\
\hline DM & 6 & 1 & El participante estaba inscrito solo en DM \\
\hline
\end{tabular}

\section{RESULTADOS}

Los datos recolectados fueron codificados temáticamente y relacionados con las preguntas de investigación. Cinco categorías emergen de este análisis: 1) "Espacios de gran aprendizaje" que se refiere al aprendizaje significativo de los contenidos específicos de cada curso, 2) El desafío de trabajar en equipo, 3) "Acercamiento a la vida real" donde se refieren al proyecto como oportunidad de acercarse a la profesión, 4) Dinámicas y tensiones en los roles profesor estudiante y 5) Motivación. En esta sección, cada una de las categorías es descrita y ejemplificada con citas de los participantes. Para ejemplificar las categorías se incluyen algunas citas textuales de los participantes, donde se denota con EG y $\mathrm{Cl}$ a los instrumentos entrevista grupal y cuestionario individual, respectivamente. El número y las siglas MED/DM corresponden al participante y el curso que estaba inscrito.

\section{Espacio de gran aprendizaje}

Los participantes indican, tanto en las entrevistas grupales como en los cuestionarios individuales, que la realización del proyecto ayudó a la comprensión y significación de los contenidos técnicos del curso. Durante el proyecto los estudiantes de MED debían realizar cálculos estructurales para los que emplearon el método de los nodos; método que demanda mucha atención en los cálculos. Las respuestas sugieren que algunos participantes pudieron comprender la importancia del método, uno de ellos menciona "el cálculo de nodos y su importancia" (Cl3-MED) como uno de los principales aprendizajes al realizar el proyecto. Similarmente, algunos participantes reconocen la importancia del cálculo de momentos como "fundamental para el cálculo de estructuras" (EG-MED).

Se mencionan como principales aprendizajes el diseño de estructuras, la utilización de programas y de impresión 3D: "adquirí conocimientos en las áreas de diseño CAD, diseño de armaduras, cálculos de cargas, el desarrollo de un informe, comunicación entre áreas, etc." (Cl7- MED-DM); "Utilizar programas ... para la creación de planos y piezas" (EG-DM); "[aprendí] distintos tipos de estructuras, diseño CAD, utilización de impresora 3D..." (Cl1-MED-DM). Los participantes valoran como aspectos positivos del proyecto la aplicación práctica del contenido teórico del curso: "el principal aprendizaje fue la aplicación directa de los métodos vistos en clase" (Cl4-MED-DM); "el que fuera una forma práctica de mostrar la materia" (Cl2-MED-DM) y "la instancia de aplicar de forma práctica conocimientos adquiridos en las clases" (CI7-MED-DM).

Por último, se mencionan aprendizajes relacionados a la comunicación y coordinación entre equipos de distintos cursos. Los participantes señalan que "el gran desafío ha sido la coordinación de los grupos en ambos cursos, es decir, el tener toda la información del diseño a tiempo para crear los planos, la interpretación nuestra cuando la información es ambigua" (EG-DM), esta cita es representativa respecto de las dificultades de coordinación entre los equipos de las distintas áreas. Para comunicar adecuadamente los requerimientos técnicos, fue necesario que propiciaran instancias de articulación entre los equipos, por ejemplo, un participante señala que "la coordinación con el equipo de dibujo fue difícil. La solución fue conversar y discutir el tema con el líder del grupo." (Cl1-MED). Pero estas instancias dieron espacio para una discusión técnica entre pares que fue valorada por los participantes "La crítica constructiva a la hora de evaluar los trabajos entre nosotros fue un espacio de gran aprendizaje" (EG-DM).

\section{El desafío de trabajar en equipo}

El trabajo en equipo fue marcado como uno de los principales desafíos, tanto en la entrevista grupal como en los cuestionarios individuales. En la entrevista grupal de MED fue referido por 6 de los 8 grupos entrevistados. Por otro lado, si bien en el cuestionario individual se presentaron dos preguntas sobre trabajo en equipo, ese asunto fue referido fuertemente en las respuestas de otras preguntas (en 15 de 28 respuestas). Según las respuestas, lo desafiante del trabajo en equipo está relacionado a: (i) cumplimiento de los plazos (ii) poca 
responsabilidad o interés de los integrantes, (iii) coordinación y organización, y (iv) respeto al contrato (instrumento usado por estudiantes de MED). Entre las citas se destaca: "Muchísimas [dificultades], por ejemplo, la coordinación y el poco interés de un integrante..." (Cl3-MED), "Cumplir con los plazos, la distribución de tareas, cumplimiento del contrato." (Cl4-MED-DM). Cabe señalar que en esta categoría se presentan los resultados del trabajo en equipo del mismo curso, no entre equipos de diferentes cursos.

Se mencionan desafíos vinculados a las dimensiones coordinación y cooperación, comunicación y resolución de conflictos del trabajo en equipo. Un grupo participante reportó que frente al incumplimiento de tareas y desinterés de uno de sus integrantes decidieron excluirlo del grupo "...La manera de manejarlo fue excluyendo al integrante que no estaba interesado" (Cl3-MED)". Esto sugiere que las estrategias que fueron entregadas en el curso para facilitar la coordinación y cooperación, como el contrato y acompañamiento de los grupos por el cuerpo docente, no fueron suficientes para guiar de manera pertinente y oportuna el trabajo en equipo. Por ejemplo, algunos de los participantes solicitan que las penalizaciones acordadas en el contrato tuvieran impacto directo en la nota, por ejemplo, "Las penalizaciones debieran repercutir en la nota de cada etapa." (Cl1-MED). En una línea similar, se propone la evaluación interna como herramienta para que los equipos influyan en las calificaciones de sus integrantes durante las diferentes etapas del proyecto: "... [que en el proyecto] debiese haber en cada etapa una nota de coevaluación y no solo al final..." (Cl1-MED).

\section{Acercamiento a la vida real}

El juego de roles planteado en el diseño de la experiencia de articulación fue valorado por los participantes como un acercamiento a la profesión. Los grupos de DM y MS actuaban respectivamente como proveedores y consultores de los grupos de MED. Específicamente, DM y MS ofrecían respectivamente servicios de dibujo técnico y cálculo estructural. En las respuestas, los estudiantes reconocieron la experiencia como un vínculo con el mundo profesional. Se identifican como desafiantes (i) la coordinación entre equipos y (ii) el manejo de incertidumbre al ser capaces de lograr el objetivo del proyecto a pesar de dificultades e imprevistos. Entre las citas se destaca: "El acercamiento a la vida real, el hecho de aplicar los cálculos y que funcione al momento de las pruebas en las maquetas...en equipo" (Cl5-MED), "El gran desafío ha sido la coordinación de los grupos en ambos cursos, es decir, el tener toda la información del diseño a tiempo para crear los planos, la interpretación nuestra cuando la información es ambigua" (EG-DM).

Adicionalmente, los participantes de DM también identificaron oportunidades de acercamiento técnico por medio de los estándares de ingeniería y espacio para la discusión técnica. Las citas destacadas son: "La creación de planos a nivel de normas internacionales y la óptima lectura de ésta"; "Normas de dibujo (las normas que deben cumplir los dibujos)" (EG-DM), "La crítica constructiva a la hora de evaluar los trabajos entre nosotros fue un espacio de gran aprendizaje" (EG-DM).

\section{Dinámicas y tensiones en los roles profesor-estudiante}

Los participantes mencionan distintos aspectos asociados al rol docente. Positivamente se reconocen las instancias de retroalimentación brindadas durante el proyecto, por ejemplo, al examinar las situaciones que favorecieron su aprendizaje durante el proyecto, un grupo indica "Los avances del informe ya que a cada entrega recibimos el feedback para mejorar, e investigar mejor el tema" (EG-MED) mientras que expresiones similares podemos encontrar en DM: "La retroalimentación del profesor a la hora de crear planos ayuda mucho para mejorar nuestras debilidades" (EG-DM).

Los participantes también señalan que necesitan que el profesor adopte roles que no fueron asumidos en este proyecto. Concretamente, (i) profesor como garante del avance del trabajo en el equipo, y (ii) rol tradicional del profesor. Con relación al primer rol, se identifican dos formas en las que esta garantía es requerida; como un fiscalizador del trabajo dentro de los equipos, por ejemplo: "Que los incisos [del contrato] se cumplan y que esto sea revisado por el docente" (Cl6-MED) y como un fiscalizador más cercano a los productos que se piden. Así, en MED se sugirió "que nos pidan más avances como grupo, a modo de progreso, para que los alumnos vayan al día con el proyecto" (CI4-MED) o, de manera más explícita, "Cuando empiezan problemas (de entrega o de malas entregas), que los profesores intenten solucionar para que no sea un problema de arrastre" (CI3-MED).

Con relación al segundo rol, algunas de las respuestas de los participantes se pueden asociar con una docencia más tradicional y un rol del estudiante menos autónomo. Por ejemplo, en uno de los grupos de entrevistas de MED se pide "Presentar [en las clases] ejemplos resueltos, explicarlos paso a paso y luego generar ejercicios para los alumnos" (EG-MED). Similarmente, otro grupo discute la necesidad de "mayores indicaciones en las etapas que aborden la mayoría de las posibilidades para que no quedemos tan en el aire" (EG3-MED). En DM se indica "creo que el profesor debería enseñarnos a usar los programas de diseño más que un tutorial que además estaba en otro idioma se aprende más practicando en clases que viendo videos, 
ya que además muchos no tenemos el programa instalado en nuestro pc". Cabe señalar que hay visiones contrapuestas en este aspecto, así por ejemplo otro de los grupos menciona el trabajo con videos como una fortaleza del curso DM indicando "En el curso de diseño en CAD hemos observado tutoriales que han permitido perfeccionar el uso de CAD a lo largo del semestre" (EG-DM).

Los participantes de MED, al reflexionar sobre sus niveles de compromiso y participación en el proyecto y el curso reconocen que debieron destinar más tiempo a las clases formales y organizarse de manera más eficiente en las distintas etapas del proyecto. Por ejemplo, "[nos faltó] ir más a clases y fortalecer el aprendizaje" (EG-MED). Pese a que las preguntas que guiaron las entrevistas grupales fueron las mismas, en DM la discusión se focalizó en sugerencias para mejorar el proyecto y no en el desempeño de los propios estudiantes en el proyecto.

\section{Motivación}

La respuesta de los estudiantes cuando cuestionados sobre la realización de un proyecto de articulación de las características mencionadas fue positiva y contundente. Recomiendan su incorporación en las ediciones futuras del curso, ya que la consideran una experiencia motivadora, entretenida y que sirve para conocer mejor a sus compañeros: "[lo recomiendo] es entretenido, práctico y una buena forma de conocerse con los nuevos compañeros" (Cl2-MED-DM); "[lo recomiendo] porque es interesante y a su vez entretenido, esto último debido a que uno ve cómo va avanzando la estructura." (CI6-MED-DM); "[lo recomiendo] porque motiva a ver de otra manera un curso y que no todo es solo teoría" (CI7-MED-DM).

Además de aumentar la motivación y entusiasmo por el curso, el proyecto se menciona como una gran herramienta para aprender contenidos importantes, entender la importancia de esos contenidos y también para desarrollar habilidades de trabajo en equipo y comunicación: "es entretenido. Además, hay que aprender a trabajar en equipo por lo que se aproxima en el futuro" (CI3-MED); "es una excelente herramienta para investigar y construir una armadura, que es un tema importante del curso, así como el trabajo en equipo." (Cl1-MED-DM). Otro aspecto motivante está relacionado al hecho de que los participantes consideran que el proyecto los acerca a su futura vida profesional: "hay un mayor interés debido a que es algo que se puede desarrollar en la práctica" (EG- MED). Por último, un estudiante menciona su alegría al ver el resultado final: "fue muy emocionante ver el producto final y someterlo a la prueba de carga. Observar la culminación del proyecto de todo un semestre en un puente fue muy valorado por mí." (CI1-MED-DM).

\section{DISCUSIÓN}

En este estudio se indagaron las percepciones estudiantiles sobre las oportunidades y desafíos a los que se vieron enfrentados al participar en una intervención que articuló curricularmente los cursos Mecánica Estática y Dinámica, Dibujo Mecánico y Mecánica de Sólidos, que corresponden al primer año de especialidad de la carrera de Ingeniería Civil Mecánica. La intervención se hizo alrededor de un proyecto de armaduras, que fue diseñado utilizando orientaciones del Aprendizaje Basado en Proyecto (ABPro). Los estudiantes valoraron esta experiencia, por cuanto les permitió situar su aprendizaje y tener un acercamiento a su profesión, lo que influyó positivamente en la motivación. Las mayores dificultades se reportaron en el trabajo en equipo y en el aprendizaje autónomo.

En la literatura hay un consenso amplio que el uso de ABPro es una estrategia flexible y adaptable para el desarrollo de las competencias profesionales (ver, Frank, et al., 2003; Karkoub, et al., 2020, Mesa et al., 2008). Sin embargo, las dificultades reportadas en el trabajo en equipo durante este proyecto parecen ser mucho mayores a las que se observan en la literatura. Por ejemplo, Karkoub et al. (2020) indican que el $10 \%$ de los grupos tuvieron dificultades, entre las que se señala el no recibir contribuciones de algunos miembros del equipo a tiempo. En nuestro caso, ese porcentaje es muy superior, al menos la mitad de los participantes se refiere al trabajo en equipo como uno de los aspectos más desafiantes.

Las medidas que se implementaron para facilitar la organización del trabajo en equipo, a saber, taller de construcción de contrato en el curso MED y coevaluación al final del proyecto, fueron insuficientes. También cabe señalar que parte de las dificultades que se reportaron en el trabajo en equipo, pueden deberse a la inexperiencia en la implementación de este tipo de metodologías colaborativas, tanto de los estudiantes (Le et al., 2018) como de los docentes que participaron en el proyecto (Frank et al., 2003). Por lo tanto, futuras versiones de la intervención debieran considerar un número mayor de estrategias focalizadas para que los participantes aprendan a trabajar colaborativamente. Además, si bien el equipo docente sostuvo un trabajo continuo y colaborativo durante el semestre para responder a las necesidades emergentes del proyecto, como lo propone la literatura (Benítez y García, 2013), se sugiere que el equipo docente se capacite en herramientas diversas para facilitar el trabajo colaborativo y, con ello, ofrecer apoyo oportuno y pertinente. 
En las entrevistas grupales, los participantes sugieren que para próximas implementaciones de este proyecto se incorporen instrumentos de coevaluación a todos los productos entregados en el proyecto. Cabe recordar que este proyecto se diseñó con sólo una instancia para la coevaluación. La sugerencia propuesta por los participantes puede interpretarse desde dos puntos de vista contrapuestos. Por un lado, que los estudiantes requieran menor autonomía; es decir, la intervención del profesor como ente regulador. Por otro lado, que requieran mayor autonomía; es decir, mayor injerencia en la evaluación, tanto en la calificación como en la crítica al desempeño de los compañeros de equipo por medio de los comentarios. En ambas interpretaciones la coevaluación sería una herramienta más para la organización y control al interior de los equipos. Independientemente de la interpretación, la inclusión periódica de instrumentos de coevaluación es aconsejable.

Cebrián-de-la-Serna et al. (2014) indican que la incorporación de este tipo de evaluaciones de manera continua permite que los estudiantes comprendan de mejor manera los criterios de evaluación. Estos autores también señalan que la coevaluación permitiría entregar herramientas para que los propios estudiantes regulen, no sólo el comportamiento de los integrantes del equipo, sino que además para que cada miembro regule de mejor manera su propio aprendizaje. En una línea similar, Bogumil y Abad (2008) sugieren que estas instancias podrían facilitar la reintegración de estudiantes con bajo rendimiento al ritmo de trabajo de sus compañeros de equipo. En relación con el número necesario de instancias propuestas para la coevaluación y el tiempo empleado para ellas no hay un acuerdo en la literatura. Por ejemplo, los autores Mercader et al. (2020) indican que 2 iteraciones de evaluación de pares junto con la retroalimentación es efectiva, mientras Wen y Tsai (2008) indican que 3 iteraciones son suficientes.

Finalmente, este estudio presenta algunas limitaciones; la valoración positiva del proyecto de armaduras puede estar sobrestimada, pues dado que la participación en el estudio es voluntaria, existe la posibilidad de que aquellos estudiantes que no se motivaron con el proyecto, tampoco hayan participado en la investigación, quedando sobre representado el grupo que valoró positivamente la experiencia. Además, los resultados de las encuestas individuales parecen ser mucho más positivos que aquellos entregados en grupos. Esto puede deberse a la temporalidad en que se hicieron las encuestas grupales e individuales, dado que las encuestas individuales se realizaron al finalizar el proyecto y las grupales 3 semanas antes. Este último periodo tiene una carga de trabajo superior para los estudiantes, por lo que las respuestas pueden estar mediadas por la presión de avanzar en el proyecto y la frustración de los grupos. Mientras que cuando respondieron la encuesta individual el curso había finalizado y el producto terminado, lo que pudo influir positivamente en la percepción del proyecto.

\section{CONCLUSIONES}

Basado en los resultados, su discusión y análisis, se puede extraer las siguientes conclusiones principales: (i) aspectos como el incremento de la motivación y aprendizaje significativo fueron reconocidos y valorados por los participantes, en concordancia con lo que se describe en la literatura; (ii) el trabajo en equipo se considera como uno de los mayores desafíos que enfrentaron en el proyecto, tanto con relación al trabajo interno del equipo (por ejemplo, coordinación y cooperación, retroalimentación y resolución de conflictos) como en la interacción con los equipos de los otros cursos (por ejemplo, coordinación y comunicación); (iii) el proyecto de armaduras descrito satisface las expectativas de los participantes y permite articular de manera adecuada los principales conocimientos técnicos de los tres cursos mencionados, generando instancias de práctica para el desarrollo de competencias técnicas, propias de cada curso, y genéricas, como el trabajo en equipo; y (iv) el proyecto puede ser mejorado con la incorporación de algunos aspectos a la propuesta, por ejemplo, la coevaluación de manera sistemática a lo largo del proyecto y capacitaciones específicas para el cuerpo docente en relación a cómo apoyar el trabajo colaborativo.

\section{AGRADECIMIENTOS}

Los autores agradecen a Carolina Matheson, Directora de Pregrado, Vicerrectoría Académica, Universidad de O'Higgins, por la discusión al inicio de la intervención y desarrollo en las entrevistas grupales.

\section{REFERENCIAS}

Baepler, P., y Walker, J. D., Active Learning Classrooms and Educational Alliances: Changing Relationships to Improve Learning, https://doi.org/10.1002/tl.20083, New Directions for Teaching and Learning, 137, 27-40 (2014)

Beneitone, P., y Esqueteni, C., Reflexiones y Perspectivas de la Educación Superior en América Latina: Informe FinalTuning, Bilbao: Universidad de Deusto - Universidad de Groningen (2007)

Benítez, A. A., y García, M. L., Un primer acercamiento al docente frente a una metodología basada en proyectos, https://doi.org/10.4067/S0718-50062013000100004, Formación Universitaria, 6(1), 21-28 (2013) 
Bogumil, F. T., y Abad, F., Mejoras del Aprendizaje y del Rendimiento de Grupos Reducidos de Estudiantes en una Asignatura de Proyectos de Ingeniería, http://dx.doi.org/10.4067/S0718-50062008000300003, Formación Universitaria, 1(3), 13-20 (2008)

Cebrián-de-la-Serna, M., Serrano-Angulo, J., y Ruiz-Torres, M., Las eRúbricas en la Evaluación Cooperativa del Aprendizaje en la Universidad, https://doi.org/10.3916/C43-2014-15, Comunicar, 43, 153-161 (2014)

Christie, M., y de Graaff, E., The Philosophical and Pedagogical Underpinnings of Active Learning in Engineering Education, https://doi.org/10.1080/03043797.2016.1254160, European Journal of Engineering Education, 42(1), 5-16 (2017)

Chua, K. J., A Comparative Study on First-Time and Experienced Project-Based Learning Students in an Engineering Design Module, https://doi.org/10.1080/03043797.2014.895704, European Journal of Engineering Education, 39(5), 556-572 (2014)

Dori, Y. J., y Belcher, J., How Does Technology-Enabled Active Learning Affect Undergraduate Students' Understanding of Electromagnetism Concepts?, https://doi.org/10.1207/s15327809jls1402_3, Journal of the Learning Sciences, 14(2), 243-279 (2005)

Frank, M., Lavy, I., y Elata, D., Implementing the Project-Based Learning Approach in an Academic Engineering Course, https://doi.org/10.1023/A:1026192113732, International Journal of Technology and Design Education, 13(3), 273-288 (2003)

Froyd, J. E., y Ohland, M. W., Integrated Engineering Curricula, https://doi.org/10.1002/j.2168-9830.2005.tb00835.x, Journal of Engineering Education, 94(1), 147-164 (2005)

Fuertes, G., Vargas, M., y otros cuatro autores, Project-based learning versus cooperative learning courses in engineering students, http://doi.org/10.1109/TLA.2015.7350066, IEEE Latin America Transactions, 13(9), 3113-3119 (2015)

Gomes, V. G., Consolidation of Engineering Education through Industrial Case Studies, International Journal of Engineering Education, 18, 4, 479-484 (2002)

Gratchev, I., y Jeng, D. S., Introducing a project-based assignment in a traditionally taught engineering course, https://doi.org/10.1080/03043797.2018.1441264, European Journal of Engineering Education, 43(5), 788-799 (2018)

Hanrahan, S. J., y Isaacs, G., Assessing Self- and Peer-assessment: The students' views, https://doi.org/10.1080/07294360123776, Higher Education Research \& Development, 20(1), 53-70 (2001)

Heitmann, G., Challenges of Engineering Education and Curriculum Development in the Context of the Bologna Process, https://doi.org/10.1080/03043790500213136, European Journal of Engineering Education, 30(4), 447-458 (2005)

Herrera, R. F., Muñoz, F. C., y Salazar, L. A., Diagnóstico del trabajo en equipo en estudiantes de ingeniería en Chile, http://dx.doi.org/10.4067/S0718-50062017000500006, Formación Universitaria, 10(5), 49-58 (2017)

Karkoub, M., Yang, CL., Karkoub, W., y Raslan, M., Undergraduate Cross-Class Research Projects for Deep Learning in Engineering Education, Advances in Engineering Education, 8(2), 28 (2020)

Krippendorff, K., Content Analysis: An Introduction to Its Methodology, 2a Ed, 3-401, Thousand Oaks, CA: Sage, ISBN 07619-1544-3, California, Estados Unidos (2004)

Le, H., Janssen, J., y Wubbels, T., Collaborative Learning Practices: Teacher and Student Perceived Obstacles to Effective Student Collaboration, https://doi.org/10.1080/0305764X.2016.1259389, Cambridge Journal of Education, 48(1), 103-122 (2018)

Mercader, C., Ion, G., y Díaz-Vicario, A., Factors Influencing Students' Peer Feedback Uptake: Instructional Design Matters, https://doi.org/10.1080/02602938.2020.1726283, Assessment \& Evaluation in Higher Education, 1(12), 11691180 (2020)

Mesa, J. M., Álvarez, J. V., Villanueva, J. M., y de Cos, F. J., Actualización de métodos de Enseñanza-Aprendizaje en Asignaturas de Dirección de Proyectos de Ingeniería, https://doi.org/10.4067/S0718-50062008000400004, Formación Universitaria, 1(4), 23-28 (2008)

Meyer, M., y Marx, S., Engineering Dropouts: A Qualitative Examination of Why Undergraduates Leave Engineering, https://doi.org/10.1002/jee.20054, Journal of Engineering Education, 103(4), 525-548 (2014)

Moore, M., Vygotsky's Cognitive Development Theory, https://doi.org/10.1007/978-0-387-79061-9_3054, In: Goldstein S., Naglieri J. A. (eds), Encyclopedia of Child Behavior and Development, Springer, Boston, MA (2011)

Partnership for 21st Century Learning (P21), Framework for 21st Century Learning Definitions, battelleforkids.org/ (2021)

Soria-Barreto, K. L., y Cleveland-Slimming, M. R., Percepción de los Estudiantes de Primer Año de Ingeniería Comercial sobre las Competencias de Pensamiento Crítico y Trabajo en Equipo, https://dx.doi.org/10.4067/S0718-

50062020000100103, Formación Universitaria, 13(1), 103-114 (2020)

Torrelles, C., Coiduras, J.L., y otros cuatro autores, Competencia De Trabajo En Equipo: Definición Y Categorización, Profr. Rev. Curric. y Form. del Profr., 15(3), 329-344 (2011)

Torrelles, C., Eina d'Avaluació de la Competència de Treball en Equip, tesis doctoral, Universitat de Lleida, España (2011) 
Vega, F., Portillo, E., Cano, M., y Navarrete, B., Experiencias de Aprendizaje en Ingeniería Química: Diseño, Montaje y Puesta en Marcha de una Unidad de Destilación a Escala Laboratorio mediante el Aprendizaje Basado en Problemas, https://doi.org/10.4067/S0718-50062014000100003, Formación Universitaria, 7(1), 13-22 (2014)

Wen, M. L., y Tsai, C., Online Peer Assessment in an In-Service Science and Mathematics Teacher Education Course, https://doi.org/10.1080/13562510701794050, Teaching in Higher Education, 13(1), 55-67 (2008)

Wertsch, J. V., Voices of the mind, A Sociocultural Approach to Mediated Action, Harvard University Press, 19-28, (1991) 